\title{
PULSED ELECTRIC FIELD FOR PASTEURIZATION OF FRESH SUGARCANE JUICE
}

\author{
Nguyen Duc Trung ${ }^{1}$, Nguyen Ngoc Hoang ${ }^{2}$, and Dang Minh Hieu ${ }^{3}$ \\ 1,2 Department of Food and Biological Process and Equipment, School of Biotechnology and Food \\ Technology, Hanoi University of Science and Technology, Hanoi, Vietnam, \\ e-mail: trung.nguyenduc@hust.edu.vn ${ }^{1}$, hoang.nguyenngoc@hust.edu.vn² \\ ${ }^{3}$ Department of Microbiology-Biochemistry-Molecular Biology, School of Biotechnology and Food \\ Technology, Hanoi University of Science and Technology, Hanoi, Vietnam, \\ e-mail: hieu.dangminh@hust.edu.vn
}

Received Date: April 19, 2017

\begin{abstract}
Pasteurization methods using pulsed electric fields (PEFs) have proved efficient at non-thermal inactivation of microorganisms and pathogens. The methods usually use a series of high intensity, narrow band electric fields that pass through target mediums where they induce irreversible damage to cellular membranes leading to the death of microorganisms by internal content loss breakage of cell homeostasis and other effects. This research proposed a simple, lab-scale PEF pasteurization equipment that uses common output electric voltages $(220 \mathrm{~V}$ and $110 \mathrm{~V}$, which provide electric power of 5.5 and $2.75 \mathrm{kV} / \mathrm{m}$, respectively) with a simple designed Human-Machine Interface control. At a voltage of $110 \mathrm{~V}$, the equipment was tested with non-sterilized Czapek medium which showed more than 1.42-log reduction in the microbial density at 30 s of the treatment and temperature below $60{ }^{\circ} \mathrm{C}$. A test on suspended E. coli in modified Czapek medium also indicated an inactivation of most of bacterial cells after 40s of the treatment. We then applied the equipment to pasteurize fresh sugarcane juice and, as a result, the content of native microorganisms in sugarcane juice was reduced 1.2-log cycle, compared to that of original juice before the treatment.
\end{abstract}

Keywords: Microorganism, Pasteurization, Pulsed electric field, Sugarcane juice

\section{Introduction}

The idea of electrical treatment of food for microbial control has been popular since the first half of the last century [1]. The process at that time utilized small, non-pulsed current, nowadays referred to as an ohmic heating process in which heat is internally generated due to the electrical resistance of materials when the electric current is passed through [2]. In this old method, heat becomes the main factor responsible for lethal effects on microorganisms. Other methods using heat transferred from water or steam are also used for food pasteurization. Although heat is an efficient means for microbial control in food, it is also responsible for changing food nature, which could cause reduction in taste, color, flavor and nutritional quality of food. Today consumers prefer fresh and natural food, thus the need for new non-thermal, efficient pasteurization methods. One of such method makes use of pulsed electric fields (PEFs) for pasteurization and possibly sterilization [3].

PEFs have been well known to cause electro-mechanical instability of the cell membrane. The cell membrane, apart from protecting the microorganism from surrounding environment, controls the passage of nutrients into the cell and end products out of the cells. Compromising membrane integrity could lead to the leakage of intracellular content, the break of cellular homeostasis and cell function [4]. 
In the fields of genetic engineering and biotechnology, a small and short pulse of electrical field can be used to induce electroporation of the cell membrane to facilitate the transfer of foreign genes into the cell. Sale and Hamilton [5] were the first to systematically study the effects of PEFs on the viability of bacteria and yeasts, proposing that the electric field induced irreversible loss of the membrane's function which was the cause of cell death. For the last few decades, PEF pasteurization implemented by application of series of high intensive, narrow band-width electrical fields has been widely studied for the inactivation of microorganisms and pathogens in various objectives from plants to juices $[6,7,8,9]$. PEFs applied to ultra-high temperature (UHT) milk and whole raw milk have showed higher efficiency in the inactivation of native microbiota, extending the milk's shelf-life significantly longer than thermally pasteurized milk [10]. Another study by Bermúdez-Aguirre and colleagues [11] on PEF pasteurization of raw milk with different treatment conditions, however, suggested minor changes in physicochemical properties, which are mainly confined to changes in the amounts of protein and fat contents. There was little efficiency in the inactivation of both mesophiles and psychrophiles in the milk, leaving a widespread issue among researchers that must be solved in the future. A study to set up conditions for application of PEFs on apple juice also demonstrated high efficiency in the inactivation of E. coli O157:H7 [12, 13]. The treatment conditions for E. coli O157:H7 were also validated with other bacteria: Grampositive (Listeria monocytogenes and Staphylococcus aureus) and Gram-negative (Salmonella enterica serovar Typhimurium). This PEF technology was shown to be an efficient substitute for conventionally thermal pasteurization, and can be operated at room temperature to retain the quality as well as heat-sensitive and valuable nutrients of foods. Nevertheless, there are still a number of technical issues of this technology that need to be overcome when scaling up for industrial applications.

Sugarcane juice is a very popular drink for refreshment in Vietnam during hot weather. However, pasteurization of sugarcane juice so far is not a common practice. The juice is usually served fresh, right after the sugarcane stalks are crushed in a crushing machine operated either by hand or electricity, and is available widely on sidewalks of many streets in Vietnam. Aside from containing several antioxidants and electrolytes, sugarcane juice contains high amounts of sugar which make it an ideal environment for the growth of microorganisms. Inactivation of native microorganisms in the juice could be possible to retain the quality, the nutrient contents, the natural states, and to increase the shelf-life of the juice. In this study, the setup conditions for PEF pasteurization of sugarcane juice were selected. PEF method was introduced as a potential solution for the inactivation of native microorganisms in fresh sugarcane juice and showed to be efficient at reducing total microorganism count in the juice. The method, in addition, showed potential as a solution of continued treatment of the juice as well as being widely applied to other juices with features of easy operation and reasonable operating cost.

\section{Materials and Experimental Design}

\section{Materials}

The medium for the cultivation and quantification of Escherichia coli was modified Czapek medium with the substitution of saccharose for glucose. The strain E. coli was an isolated strain and provided by the Laboratory of Microbiology-Biochemistry-Molecular Biology, School of Biotechnology and Food Technology, Hanoi University of Science and Technology. 
Medium for the quantification of microorganisms in sugarcane juice was Czapekagar medium. Table 1 provides description of the mediums used in this study.

Table 1: Description of Mediums for Microbial Growth

\begin{tabular}{lllll}
\hline \multirow{2}{*}{ Contents } & \multicolumn{4}{l}{ Quantities of contents for 1 liter of medium (g/l) } \\
\cline { 2 - 5 } & $\begin{array}{l}\text { Czapek } \\
\text { (liquid) }\end{array}$ & $\begin{array}{l}\text { Czapek-agar } \\
\text { (solid) }\end{array}$ & $\begin{array}{l}\text { Modified } \\
\text { Czapek } \\
\text { (liquid) }\end{array}$ & $\begin{array}{l}\text { Modified } \\
\text { Czapek-agar } \\
\text { (solid) }\end{array}$ \\
\hline Saccharose & 30 & 30 & - & - \\
Glucose & - & - & 5 & 5 \\
$\mathrm{NaNO}_{3}$ & 0.3 & 0.3 & 0.3 & 0.3 \\
$\mathrm{~K}_{2} \mathrm{HPO}_{4}$ & 0.1 & 0.1 & 0.1 & 0.1 \\
$\mathrm{KCl}$ & 0.5 & 0.5 & 0.5 & 0.5 \\
$\mathrm{MgSO}_{4}$ & 0.5 & 0.5 & 0.5 & 0.5 \\
$\mathrm{FeSO}_{4} .7 \mathrm{H}_{2} \mathrm{O}$ & 0.01 & 0.01 & 0.01 & 0.01 \\
Agar & - & 20 & - & 20 \\
\hline
\end{tabular}

- Well dissolved in 1 liter of deionized water.

- Sterilized at $121^{\circ} \mathrm{C}, 20 \mathrm{~min}$ in an Upright Autoclave HV-150 machine (Hirayama, Japan).

- Adjusted to $\mathrm{pH} 7$ and stored at $4^{\circ} \mathrm{C}$ in refrigerator.

\section{Experimental Setup for PEF Equipment}

The design of experimental PEF pasteurization equipment at laboratory scale is described in Figure 1. Fresh sugarcane juice was placed in treatment chamber which has dimensions (mm) of $150 \times 40 \times 30$ (Length $\times$ Width $\times$ Height). PEF pasteurization equipment was supplied by an ACS (Alternate Current Source) from electrical network (220VAC/50Hz). The electrical field intensity (E) was changed by voltage regulation which was implemented by the power transformer with variable voltage at the output. The direction of electric field (E) was changed continuously with the frequency of $50 \mathrm{~Hz}$ (Figure $1 \mathrm{~A}$ ) which applied on the two electrodes (ME1 and ME2). The alternative changes of the polarization on cells induces irreversible damage on cell membranes, thus causing microbial inactivation.

The output voltage (U) from the transformer could be set to: $220 \mathrm{~V}, 110 \mathrm{~V}$ or $100 \mathrm{~V}$. The electric field was passed through sugarcane juice in treatment chamber between the two thin metallic electrodes (ME1 and ME2). The distance (d) between ME1 and ME2 was $40 \mathrm{~mm}(0.04 \mathrm{~m})$ so that the corresponding values of the electrical field intensity $(\mathrm{E}=\mathrm{U} / \mathrm{d})$ can be set to $5.5 \mathrm{kV} / \mathrm{m}, 2.75 \mathrm{kV} / \mathrm{m}$ or $2.5 \mathrm{kV} / \mathrm{m}$. The frequency of output voltage and the electrical field were always constant, and so was the frequency of electrical network $(50 \pm 0.1 \mathrm{~Hz})$.

The commands to the driver circuit was sent from the computer via the NIMyRIO-1900 card (National Instruments, USA). The driver circuit included the triac BTA20-20A-600V which played an important role in exact interval time control of the PEF process. The control signal was sent to control gate of the triac via an opto-coupling device to ensure the operational safety for the NIMyRIO-1900 card. The detailed diagram can be found in figure 1B. 

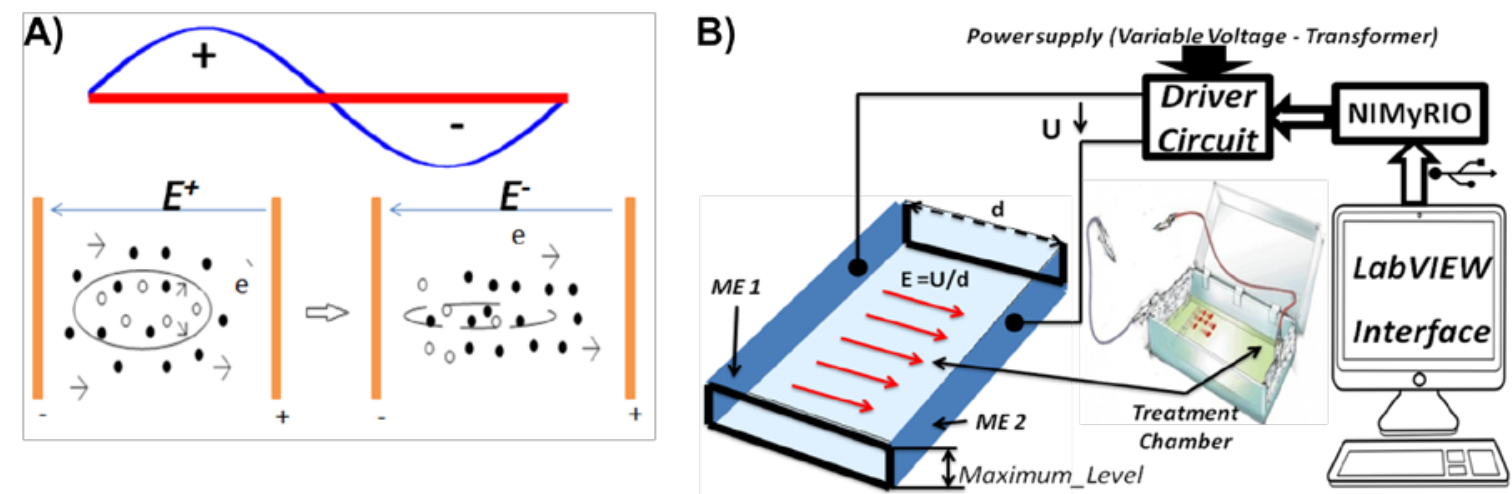

Figure 1. Experimental principle of PEF pasteurization. A) The principle of ACS PEF with two polarizations. B) A diagram of PEF pasteurization equipment at laboratory scale.

The pasteurization system was operated via custom-designed Human Machine Interface (HMI) which was programmed in LabVIEW (Laboratory Virtual Instrumentation Engineering Workbench) environment, version 2014 (National Instrument, USA) (Figure 2). The pasteurization time could be preset via the "Numeric Control" box on the HMI after the source was connected to circuit driver and before the OFF/ON button switch was turned to the "ON" status. The pasteurization process was automatically stopped by the control device after preset interval time for each experiment.

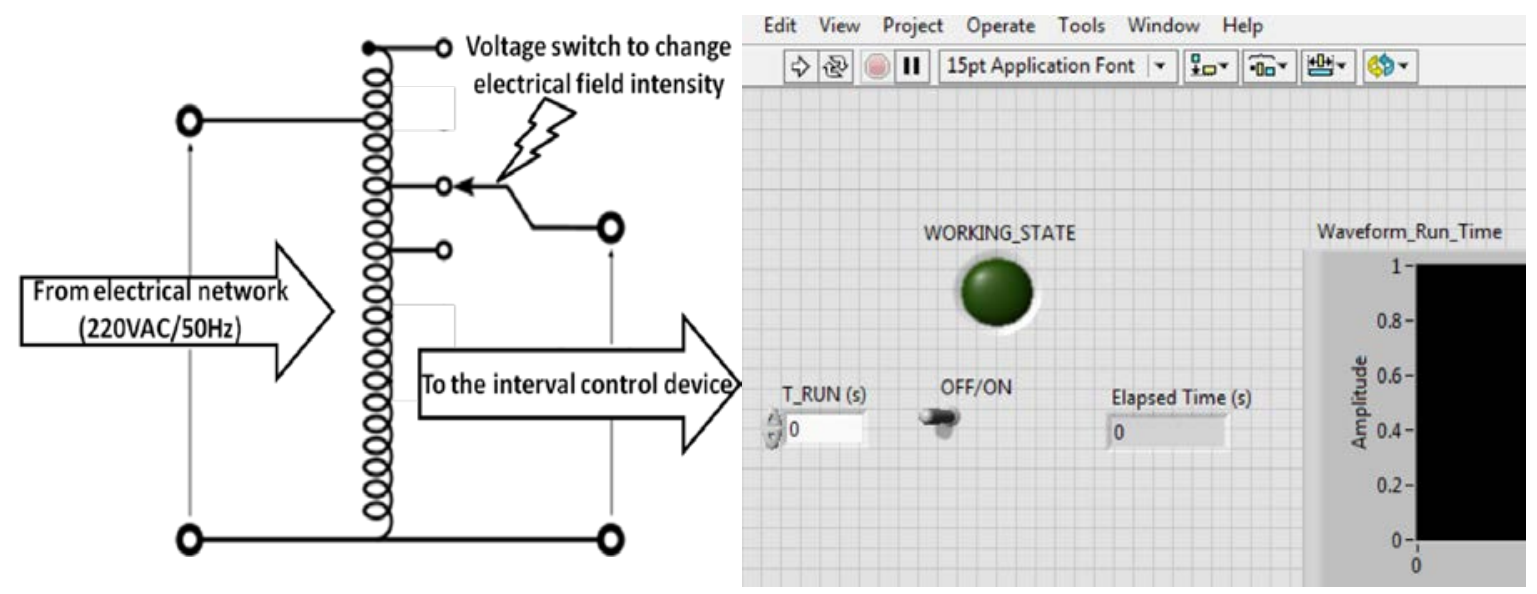

Figure 2. Variable voltage source to change electrical field intensity and Human-Machine Interface to control pasteurization interval time via computer.

\section{PEF of Sugarcane Juice and Quantitative Measurements of E. coli and Sugarcane Juice's Microorganisms}

A modified Czapek medium containing suspended E. coli cells at a density of $10^{4}$ cells $/ \mathrm{ml}$ (or fresh sugarcane juice) was filled up the treatment chamber up to maximum $3 / 4$ of the volume of the chamber. Petri dishes containing modified Czapek-agar medium and Czapek-agar medium for the cultivation of $E$. coli and native microorganisms of sugarcane juice, respectively, were prepared in advance. After PEF treatment, $100 \mu \mathrm{l}$ of E. coli suspension (or sugarcane juice) was taken out by micropipette and placed on prepared petri dish where it was distributed equally over the medium's surface. 
Each treatment was repeated at least three times. The dishes were then placed in the incubator at $37^{\circ} \mathrm{C}$ for 2 days for the formation of microbial colonies. The number of colonies formed in each dish was counted and the final microorganism density was tenfolds of that number (CFU-colony forming unit/ml).

\section{Results and Discussion}

\section{Effect of PEF Pasteurization's Treatment Time on Temperature and pH of Medium}

It has been well known that when passing through mediums, electric fields will induce heat due to the internal resistances of the mediums. Conventional pasteurization methods using electric fields thus are referred to as ohmic pasteurization. In our experiments, we wanted to separate the effects of pulsed electric fields and heat for preventing heat-induced loss of valuable contents in the mediums. Therefore, treatment conditions should be selected in order to control the temperature of mediums below $60^{\circ} \mathrm{C}$, the temperature that most of microorganisms can still tolerate. We tested on modified Czapek medium with two output electric voltages: $220 \mathrm{~V}$ and $110 \mathrm{~V}$, which correspond to the two powers: 5.5 and 2.75 $\mathrm{kV} / \mathrm{m}$, respectively. Treatments was conducted up to 12 and $40 \mathrm{~s}$ for the voltages of $220 \mathrm{~V}$ and $110 \mathrm{~V}$, respectively. Figure 3 indicates the fluctuations of the temperature and $\mathrm{pH}$ of the medium while treating with the two voltages. It showed that the medium's $\mathrm{pH}$ was not changed too much and was kept between 6 and 7 in both cases, while the temperature of the medium gradually increased. In the case of $220 \mathrm{~V}$, the temperature increased quickly after $5 \mathrm{~s}$ and went over $60^{\circ} \mathrm{C}$ at $9 \mathrm{~s}$. The temperature in the case of $110 \mathrm{~V}$, on the other hand, slowly increased during the first $20 \mathrm{~s}$ and reached $55^{\circ} \mathrm{C}$ at $30 \mathrm{~s}$. After $30 \mathrm{~s}$ of treatment, the temperature of the medium increased quickly to $64^{\circ} \mathrm{C}$ at $35 \mathrm{~s}$. Considering the output power and the ease and convenience of system control, we chose the output electric voltage of $110 \mathrm{~V}$ with treatment time up to $30 \mathrm{~s}$ for our next experiments.

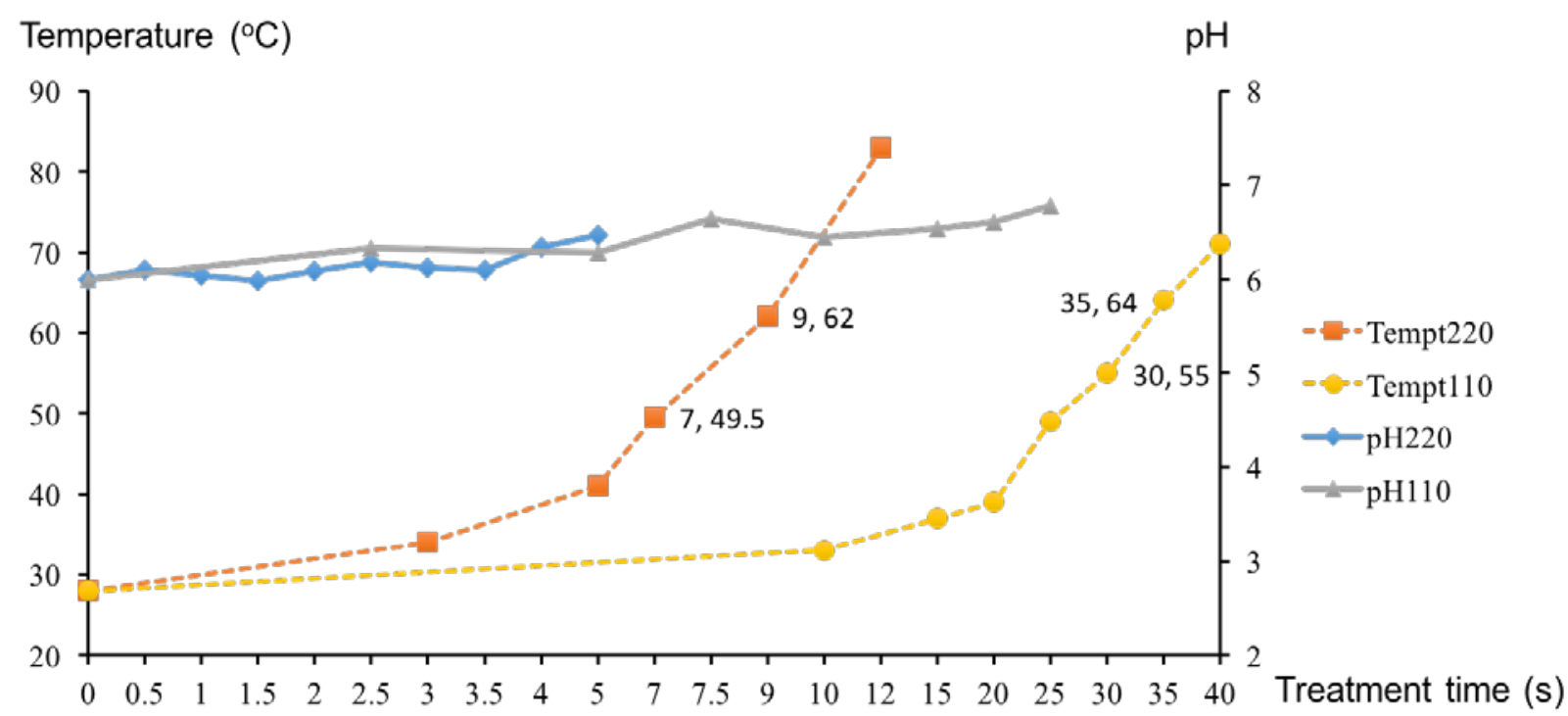

Figure 3. Effect of the power of electric pulses and treatment time on the physical conditions of liquid medium. Tempt220, tempt110 and pH220, pH110 denote fluctuations in temperature and $\mathrm{pH}$ of the medium while pulsed at the voltages of $220 \mathrm{~V}$ and $110 \mathrm{~V}$, respectively. 
In order to validate the above setup conditions for PEF pasteurization, nonsterilized Czapek medium was used. At certain time points during the PEF treatment, 100 $\mu \mathrm{l}$ of medium was taken out from the treatment chamber to perform quantifications of total microorganisms following the procedure described in previous section. As indicated in Figure 4, PEF pasteurization was effective in inactivation of microorganisms in nonsterilized Czapek medium. At $30 \mathrm{~s}$ of PEF treatment where the medium's temperature is still below $60^{\circ} \mathrm{C}$, the microbial content reduced 1.42-log cycle compared to non-treated medium. PEF pasteurization for $40 \mathrm{~s}$ could inactivate almost microorganisms of the medium.

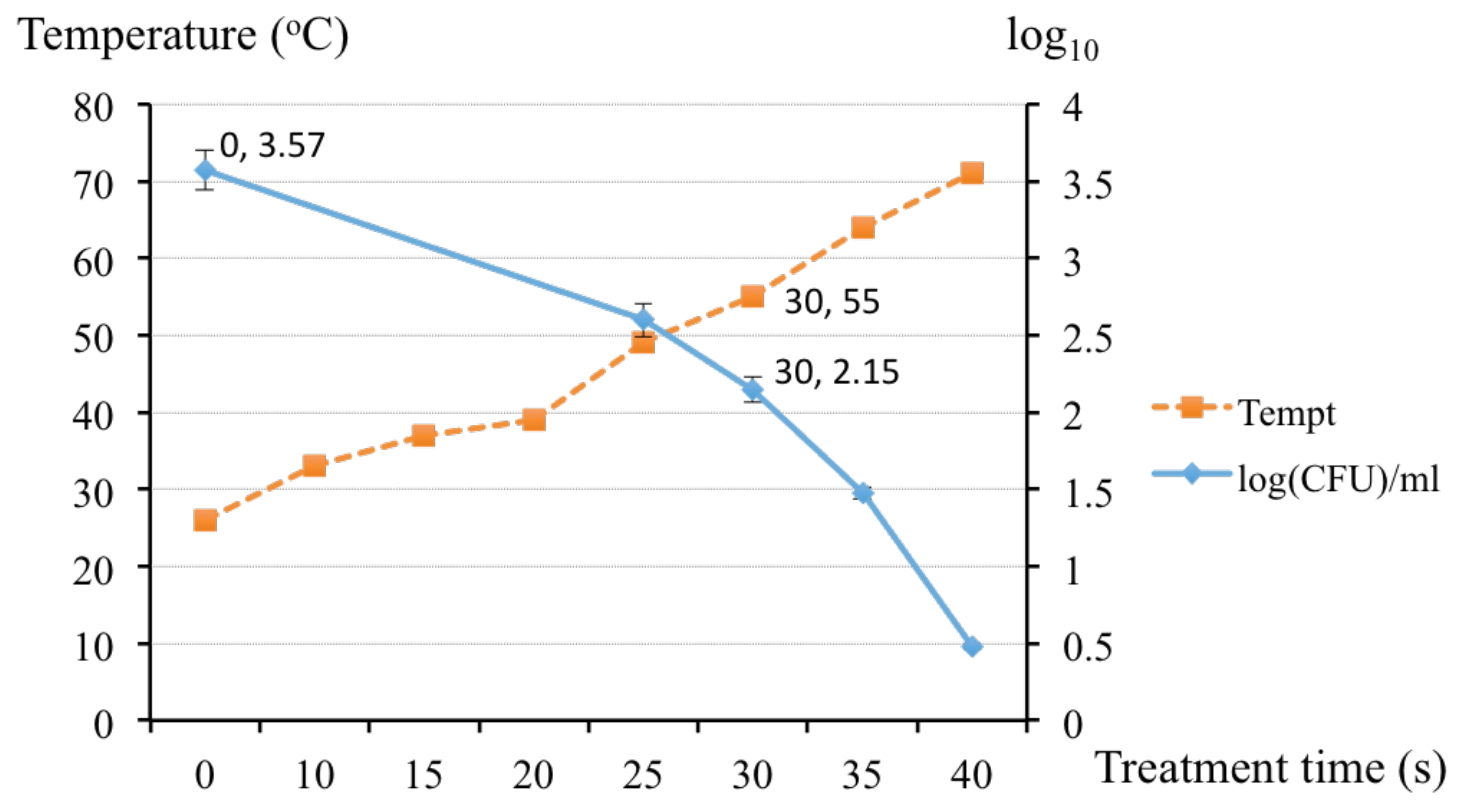

Figure 4. Fluctuations of temperature and microbial content with time, in non-sterilized Czapek liquid medium during PEF pasteurization. Data presented as mean \pm standard deviation.

\section{Inactivation of Suspended E. coli in Modified Czapek Medium}

In this experiment, we tested the efficiency of our PEF pasteurization equipment in the inactivation of the bacteria E. coli suspended in modified Czapek medium, where glucose was used as a substitute for saccharose, providing better use of $E$. coli for proliferation. The liquid medium was prepared and sterilized in advance at $121^{\circ} \mathrm{C}, 20 \mathrm{~min}$. After it was cooled down to room temperature, E. coli cells were added into the medium to the density of $10^{4}$ cells $/ \mathrm{ml}$. The suspension was then filled in the treatment chamber for PEF pasteurization. Cell counting was performed at different time points following quantitative procedure for bacterial content described above. The data shown in Figure 5 suggests that our PEF equipment and the setup conditions were efficient in removing most of $E$. coli cells in the medium at the temperature lower than $60^{\circ} \mathrm{C}$. It showed a higher efficiency in the reduction of microbial content in this experiment, compared to previous experiment with non-sterilized Czapek medium. A possible explanation for this case is that the medium contained only $E$. coli while the medium in previous case contained wide range of microorganisms including fungus, yeasts and other bacteria. Due to differences in membrane structure of cells, some may be more susceptible to PEFs than others. 


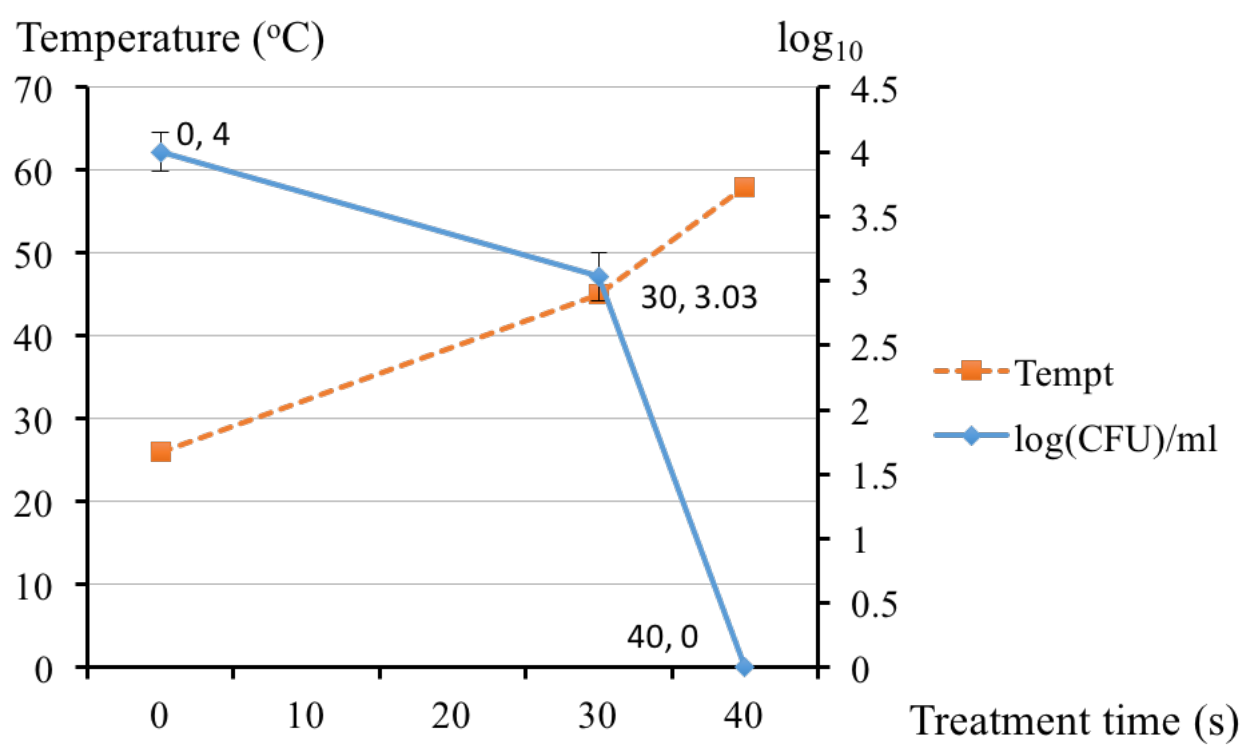

Figure 5. The inactivation of suspended E. coli in modified Czapek medium by PEF pasteurization. Data present as mean \pm standard deviation.

A previous report by Zgalin and colleagues [14] on the effect of microsecond and nanosecond pulsed electric fields on the inactivation of E. coli in water samples also suggested a reduction in bacterial counts when using microsecond pulses, but not any detectable effect with nanosecond pulses. The reduction in bacterial count was only achieved when nanosecond and microsecond pulses were combined. It should be noted that, in most previous cases of study with PEF pasteurization, high intensity (3 - 30 $\mathrm{kV} / \mathrm{cm}$ ) and very short pulses were applied which might require high investment in equipment for pulse generation and control. The system used in our study is considered a simple setup which provided efficient inactivation of $E$. coli in bacterial culturing medium, thus indicating an advantage for a wide-range of applications.

\section{Inactivation of Native Microorganisms in Fresh Sugarcane Juice}

To prove the efficiency of the PEF equipment with natural mediums, we tested the system with fresh sugarcane juice. The treatment chamber was filled with sugarcane juice up to $3 / 4$ of the maximum volume of the chamber. An electric field with the voltage of $110 \mathrm{~V}$ was passed through the juice in the chamber producing an electric power of $2.75 \mathrm{kV} / \mathrm{m}$. At certain time points during the treatment, total microorganisms were counted, following the quantitative procedure described in previous section. As shown in Figure 6, for sugarcane juice, the PEF treatment could be prolonged to $70 \mathrm{~s}$ while the juice's temperature was still kept below $60^{\circ} \mathrm{C}$. At $70 \mathrm{~s}$ of the treatment, the native microbial content was reduced 1.2log cycle, compared to that of the original juice before treatment. This efficiency was lower than that in the $E$. coli case.A possible explanation could be that the microbial structure of sugarcane juice contains different types of microorganisms including fungus, yeasts and bacteria, which express different susceptibilities to PEFs. Nevertheless, the proposed PEF pasteurization equipment was efficient at the inactivation of native microorganisms in fresh sugarcane juice. Controlling the temperature of the juice by submerging $3 / 4$ of the chamber in cold water could prolong the time of treatment for higher efficiency in reduction of microbial growth of the juice. 


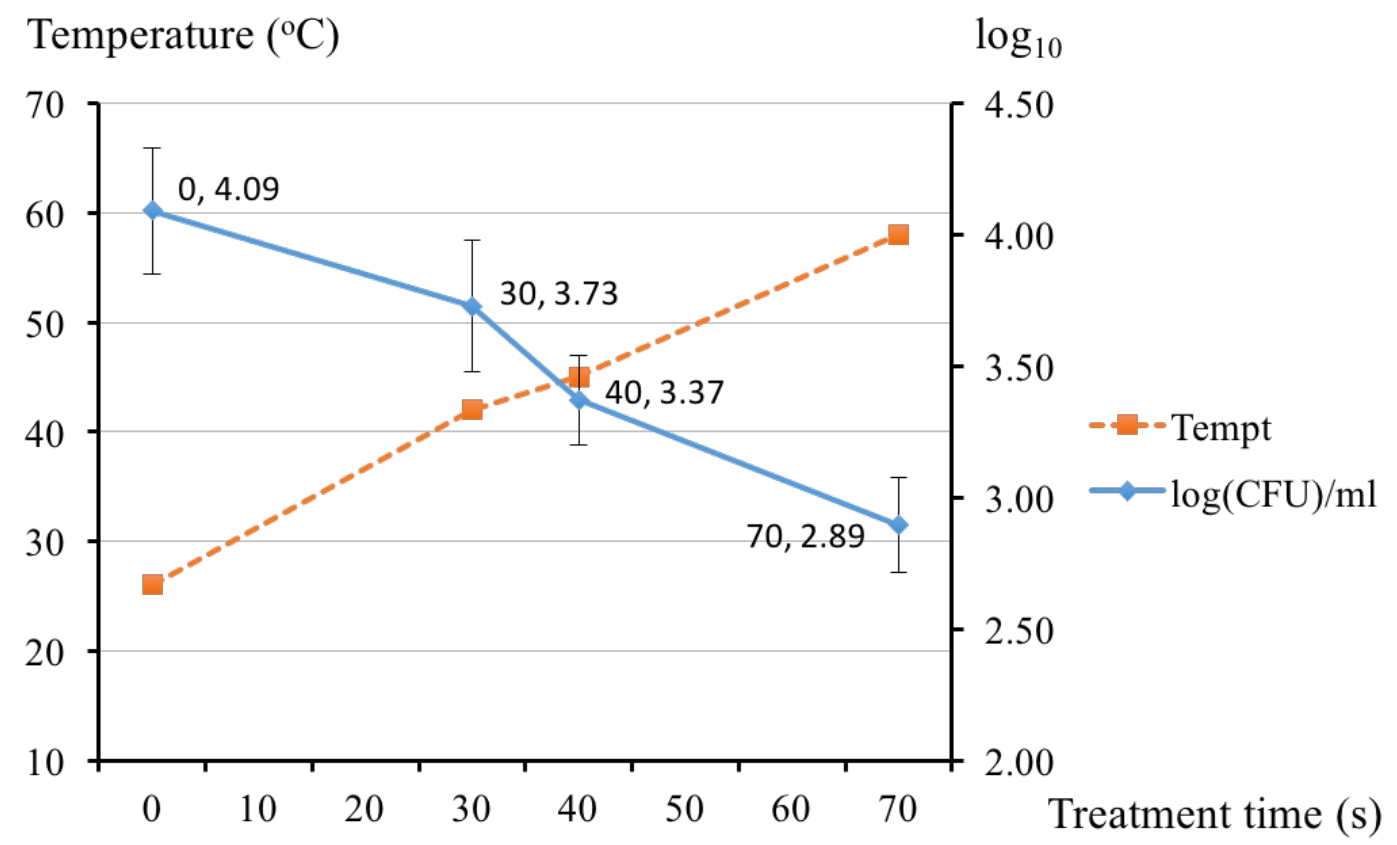

Figure 6. The inactivation of native microorganisms in fresh sugarcane juice by PEF pasteurization. Data present as mean \pm standard deviation.

\section{Conclusions}

We proposed a lab-scale PEF pasteurization equipment that is simple, utilizes common output electric voltages ( $220 \mathrm{~V}$ and $110 \mathrm{~V})$, with easy-to-find spare parts and materials, and a simple designed Human-Machine Interface control. The equipment has proved efficient in the inactivation of suspended $E$. coli in modified Czapek medium, and native microorganisms in Czapek medium and fresh sugarcane juice. Although there are still many technical issues that need to be overcome to improve the efficiency and to bring the system to higher scale, the study suggests the potential of this equipment for small scale or family applications. In the future, we are going to study the design of a compact system, which possibly allows continued treatment of fluids as well as finding suitable treatment conditions for different juices.

\section{References}

[1] S. Palaniappan, S.K. Sastry, and E.R. Richter, "Effects of electricity on microorganism: A review,” Journal of Food Processing and Preservation, Vol. 14, No. 5, pp. 393-414, 1990.

[2] N. Kaur, and A.K. Singh, "Ohmic Heating: Concept and Applications-A review," Critical Reviews in Food Science and Nutrition, Vol. 56, pp. 2338-2351, 2016.

[3] S. Jeyamkondan, D.S. Jayas, and R.A. Holley, "Pulsed Electric Field Processing of Foods: A review,” Journal of Food Protection, Vol. 62, No. 9, pp. 1088-1096, 1999.

[4] B.-L. Qin, U.R. Pothakamury, G.V. Barbosa-Cánovas, B.G. Swanson and D.M. Peleg, "Nonthermal pasteurization of liquid foods using high-intensity pulsed electric fields," Critical Reviews in Food Science and Nutrition, Vol. 6, No. 6, pp. 603-627, 1996. 
[5] A.J.H. Sale and W.A. Hamilton, "Effects of high electric fields on microorganism: I. killing of bacteria and yeasts," Biochimia et Biophysica Acta (BAA) - General Subjects, Vol. 148, No. 3, pp. 781-788, 1967.

[6] W. Sitzmann, E. Vorobiev, and N. Lebovka, "Application of electricity and specifically pulsed electric fields in food processing: Historical backgrounds," Innovative Food Science and Emerging Technologies, Vol. 37, pp. 302-311, 2016.

[7] F.J. Barba, O. Parniakov, S.A. Prreira, A. Wiktor, N. Grimi, N. Boussetta, J.A. Saraiva, J. Raso, O. Martin-Belloso, D. Watrowa-Rajchert, N. Lebovka, and E. Vorobiev, "Current applications and new opportunities for the use of pulsed electric fields in food science and industry,” Food Research international, Vol. 77. Pp. 773-789, 2015.

[8] R.A.H. Timmermans, M.N.N. Groot, A.L. Nederhoff, M.A.J.S. van Boekel, A.M. Matser and H.C. Mastwijk, "Pulsed electric field processing of different fruit juices: Impact of $\mathrm{pH}$ and temperature on inactivation of spoilage and pathogenic microorganisms,” International Journal of Food Microbiology, Vol. 173, pp. 105-111, 2014.

[9] G. Saldanã, I. Álvarez, S. Condón, and J. Raso, “Microbiological Aspects Related to the Feasibility of PEF Technology for Food Pasteurization," Critical Reviews in Food Science and Nutrition, Vol. 54, pp. 1415-1426, 2014.

[10] M. Walkling-Ribeilo, F. Noci, D.A. Cronin, J.G. Lying and D.J. Morgan, "Antimicrobial effect and shelf-life extension by combined thermal and pulsed electric field treatment of milk," Journal of Applied Microbiology, Vol. 106, pp. 241-248, 2009.

[11] D. Bermúdez-Aguirre, S. Fernández, H. Esquivel, P.C. Dunne and G.V. BarbosaCánovas, "Milk Processed by Pulsed Electric Fields: Evaluation of microbial quality, physicochemical characteristics, and selected nutrients at different storage conditions", Journal of Food Science, Vol. 76, No. 5, pp. 289-299, 2011.

[12] G. Saldanã, E. Puértolas, S. Monfort, J. Raso, and I. Álvarez, "Defining treatment conditions for pulsed electric field pasteurization of apple juice,” International Journal of Food Microbiology, Vol. 151, pp. 29-35, 2011.

[13] Z.A. Valappil, X. Fan, H.Q. Zhang and R.L. Rouseff, "Impact of thermal and nonthermal processing technologies on unfermented apple cider aroma volatiles," Journal of Agriculture and Food Chemistry, Vol. 57, pp. 924-929, 2009.

[14] M.K. Zgalin, D. Hodzíc, M. Rebersek and M. Kanduser, "Combination of microsecond and nanosecond pulsed electric field treatments for inactivation of Escherichia coli in water samples,” Journal of Membrane Biology, Vol. 245, pp. 643650, 2012. 